\section{Nachhaltiger Verkehr schafft wirtschaftliche Chancen}

\author{
Der Verkehrssektor ist derzeit für etwa $20 \%$ der Treibhausgas- \\ emissionen Deutschlands verantwortlich. Da durch die Energie- \\ wende die erneuerbaren Energien im Zentrum des künftigen \\ Energiesystems stehen werden, ist der Ausbau der Elektromobilität \\ der zukünftige Wachstumspfad. Welche Potenziale bieten sich \\ der Branche? Von Claudia Kemfert
}

U $\mathrm{m}$ das Pariser Abkommen für mehr Klimaschutz umzusetzen, muss der Verkehrssektor konsequent auf Klimaschutz und Nachhaltigkeit ausgerichtet werden. Während in anderen Sektoren seit 1990 Emissionsminderungen erzielt wurden, sind die Emissionen des Verkehrs im gleichen Zeitraum leicht angestiegen. Der größte Teil der Treibhausgasemissionen stammt dabei aus dem Straßenverkehr. Dieser trägt zudem wesentlich zur Feinstaubbelastung bei.

Ein zentrales Element eines klimagerechten und nachhaltigen Verkehrssystems muss die Verringerung des motorisierten Individualverkehrs sowie die Stärkung intelligenter und integrierter Mobilitätslösungen sein. Dabei können eine Verkehrsvermeidung und Verlagerung auf Schiene, ÖPNV, Rad- sowie Fußverkehr die Emission von Treibhausgasen und den Energieverbrauch verringern sowie weitere Probleme des Verkehrs wie Flächenverbrauch, Lärm und Unfallrisiken berücksichtigen.

\section{Elektrifizierung des Verkehrs}

Eine direkte Elektrifizierung des Verkehrs mit erneuerbaren Energien ist technologisch und wirtschaftlich effizient und mit einem realisierbaren Zubau der Anlagen vereinbar. Die Einbindung von Batterien kann systemdienlich zur Stromspeicherung und Entlastung der dezentralen Netze beitragen. Der Güterverkehr kann ebenso mit elektrischen Lkws oder aber direkt auf der Schiene stattfinden. Für lange Distanzen bieten sich flüssige Treibstoffe an, die aus erneuerbaren Energien gewonnen werden, wie beispielsweise Power to Gas. Insbesondere für den Schiffsverkehr ist dies relevant. Der großflächige Einsatz von Power to Gas oder Wasserstoff für alle Verkehrsbereiche würde einen bis zu siebenfachen Mehrausbau erneuerbarer Energien nach sich ziehen. Und es bedarf einer adäquaten Infrastruktur, die heute errichtet werden muss. „Technologieoffenheit" kann zu erheblichen Fehlentwicklungen führen, welche verlorene Erstinvestitionen (sunk investments) nach sich ziehen. Eine gezielte Technologieförderung ist sinnvoll, da ein vermeintlich neutraler Regulierungsrahmen angesichts der privilegierten Ausgangslage des Verbrennungsmotors die neuen Technologien benachteiligen würde. Auch mit Blick auf die zu schaffenden Infrastrukturen sollten diejenigen Technologien gefördert werden, die langfristig die Einhaltung der Klimaziele gewährleisten. Mehrere unterschiedliche Infrastrukturen aufzubauen, ist teuer und ineffizient.

Die Elektromobilität ist ein zentraler Baustein der nachhaltigen Verkehrswende. Aufgrund des sehr hohen Wirkungsgrads ist sie besonders geeignet, die Klima- und Umweltauswirkungen des Verkehrs grundlegend zu verringern. Zudem emittieren elektrische Antriebe lokal keine Schadstoffe. Elektroautos sind allerdings nur dann sinnvoll, wenn sie mit erneuerbaren Energien kombiniert werden und Teil einer konsequent auf Nachhaltigkeit ausgerich- teten Verkehrspolitik sind. Gerade in Großstädten spielt neben dem ÖPNV auch der Individualverkehr eine erhebliche Rolle. In der Zukunft werden zwei Drittel der Menschheit in Ballungsräumen mit mehr als einer Million Einwohner leben. Nachhaltige „Mobilitätsdienstleistungen" wie auch Carsharing werden gerade in Ballungsräumen an Bedeutung gewinnen.

\section{Chancen für die Volkswirtschaft}

Deutschland sollte die erneuerbaren Energien deutlich schneller als bisher ausbauen. Außerdem sollte sich die deutsche Regierung für strengere Grenzwerte in der Europäischen Union einsetzen. Statt eine Kaufprämie für Elektroautos einzuführen, ohne aber die Mobilität als Ganzes auf Nachhaltigkeit auszurichten, sollte besser eine Quote für neu zugelassene Fahrzeuge von $25 \%$ ab dem Jahr 2025 eingeführt werden. Zudem wird noch immer ausgerechnet der umweltschädlichste Treibstoff, der Diesel, indirekt subventioniert. Es wäre sinnvoll, die Steuererleichterung abzuschaffen und die Dieselsteuer zumindest auf das Niveau der Benzinsteuer anzuheben. Dies würde dem Staat Einnahmen von acht Milliarden Euro pro Jahr verschaffen. Diese können verwendet werden, um die Ladeinfrastruktur auszubauen und den Schienenverkehr zu stärken.

Die wirtschaftlichen Chancen einer nachhaltigen Mobilität sind für die deutsche Volkswirtschaft enorm. Die Märkte gehören denen, die sie sehen. Und wer nicht mit der Zeit geht, geht mit der Zeit. Es muss dringend umgesteuert werden.

AUTORIN + KONTAKT

Dr. Claudia Kemfert leitet seit 2004 die Abteilung Energie, Verkehr, Umwelt am Deutschen Institut für Wirtschaftsforschung (DIW Berlin) und ist seit 2009 Professorin für Energieökonomie und Nachhaltigkeit an der Hertie School of Governance (HSoG).

Deutsches Institut für Wirtschaftsforschung e. V. (DIW Berlin), Mohrenstr. 58, 10117 Berlin. Tel.: +4930 89789-663, E-Mail: sekretariat-evu@diw.de Website: https://www.diw.de/deutsch 